\title{
Superconductivity-enhanced bias spectroscopy in carbon nanotube quantum dots
}

Grove-Rasmussen, Kasper; Jørgensen, Henrik Ingerslev; Andersen, Brian Møller; Paaske, Jens; Jespersen, Thomas Sand; Nygård, Jesper; Flensberg, Karsten; Lindelof, Poul Erik

Published in:

Physical Review B Condensed Matter

DOI:

10.1103/PhysRevB.79.134518

Publication date:

2009

Document version

Early version, also known as pre-print

Citation for published version (APA):

Grove-Rasmussen, K., Jørgensen, H. I., Andersen, B. M., Paaske, J., Jespersen, T. S., Nygård, J., Flensberg, K., \& Lindelof, P. E. (2009). Superconductivity-enhanced bias spectroscopy in carbon nanotube quantum dots. Physical Review B Condensed Matter, 79(13), 134518. https://doi.org/10.1103/PhysRevB.79.134518 


\title{
Superconductivity-enhanced bias spectroscopy in carbon nanotube quantum dots
}

\author{
K. Grove-Rasmussen, * H. I. Jørgensen, B. M. Andersen, J. Paaske, T. S. Jespersen, J. Nygård, \\ K. Flensberg, and P. E. Lindelof \\ Nano-Science Center, Niels Bohr Institute, University of Copenhagen, Universitetsparken 5, 2100 Copenhagen Ø, Denmark
}

(Received 30 January 2009; revised manuscript received 11 March 2009; published 21 April 2009)

\begin{abstract}
We study low-temperature transport through carbon nanotube quantum dots in the Coulomb blockade regime coupled to niobium-based superconducting leads. We observe pronounced conductance peaks at finite sourcedrain bias, which we ascribe to elastic and inelastic cotunneling processes enhanced by the coherence peaks in the density of states of the superconducting leads. The inelastic cotunneling thresholds display a marked dependence on gate voltage caused by different tunneling renormalizations of the two subbands in the nanotube. Finally, we discuss the gate-dependent subgap structure observed in a strongly coupled device with odd electron occupation.
\end{abstract}

DOI: 10.1103/PhysRevB.79.134518

PACS number(s): 73.21.La, 73.23.Hk, 73.63.Fg, 74.50.+r

Superconducting electrodes provide a useful means of sharpening the spectroscopic features observed in tunneling experiments. In the superconducting phase, an otherwise nearly constant density of states (DOS) acquires a gap of width $2 \Delta$ centered at the Fermi level and characteristic sharp coherence peaks at the gap edges $\pm \Delta$. These peaks transform a featureless metallic electrode into a high-resolution tunneling probe. This widely used investigative too ${ }^{1}$ was demonstrated already by Giaever's seminal work ${ }^{2}$ from 1960 and more recently used to obtain a high-resolution bias spectrum of the levels in a metallic Al nanoparticle. ${ }^{3}$

Here we report low-temperature transport measurements in which this type of BCS focusing promotes an otherwise featureless elastic (EL) cotunneling conductance to sharp peaks at bias voltages $V_{\text {sd }}= \pm 2 \Delta / e$, corresponding to the onset of quasiparticle (QP) cotunneling. In the same way, inelastic (INEL) cotunneling processes involving transitions between two subbands in the nanotube are revealed as sharp peaks rather than steps or cusps in the nonlinear conductance. This sharpening of cotunneling lines inside the Coulomb diamonds allows us to investigate more closely the tunneling-induced gate voltage dependence of the orbital splitting. ${ }^{4}$ Finally, we discuss an unusual subgap structure observed in a particularly well-coupled device signaling the importance of both multiple Andreev reflections (MARs) and dynamically generated bound states in spinful dots.

A number of experiments have already investigated interesting aspects of quantum dots with superconducting electrodes, such as supercurrent, ${ }^{5-7} \mathrm{MAR},{ }^{8}$ and effects of size and charge quantization in the Fabry-Pérot, ${ }^{9-12}$ Kondo, ${ }^{13-18}$ and Coulomb blockade regimes. ${ }^{14,19-22}$ We present measurements performed on two different single-walled carbon nanotube (SWCNT) quantum dots coupled to niobium-based superconducting leads. ${ }^{7,8}$ Our first device (device A) is poorly coupled to the leads and shows regular Coulomb blockade diamonds with clear onset of quasiparticle (elastic cotunneling) current. The second device (device B) is more strongly coupled to the leads and displays a fourfold degenerate shell structure with both elastic and inelastic cotunneling lines which are sharpened by the superconducting leads.

The devices are made by growing high quality SWCNT by chemical vapor deposition from predefined catalyst islands. ${ }^{10,15}$ Contacts are made of Nb-based trilayers, $x / \mathrm{Nb} / x$ (about 5/60/10 nm), with $x=\mathrm{Pd}, \mathrm{Ti}$, where $x$ is thermally evaporated and the $\mathrm{Nb}$ is deposited by sputtering technique. The superconducting trilayers are tested via four terminal devices on the same chip and show transition temperatures close to $T=9 \mathrm{~K}$. Nevertheless, the actual gap at the nanotube indicates a critical temperature of $T_{c} \approx 1.7 \mathrm{~K}$, which might be related to the formation of $\mathrm{NbO}\left(T_{c} \sim 1.4 \mathrm{~K}\right)$ (Ref. 23) or contamination of the lower $\mathrm{Nb} / x$ interface. Samples from three different processing rounds revealed similarly reduced $T_{c}$ in transport measurements. However, the high quality of the presented measurements is a promising first step toward Nb-based SWCNT Josephson junctions.

Figure 1(a) shows the conductance versus gate, and bias voltage (bias spectroscopy plot) at $T=0.3 \mathrm{~K}$ for device A consisting of a carbon nanotube quantum dot coupled weakly to $\mathrm{Ti} / \mathrm{Nb} / \mathrm{Ti}$ leads. It reveals more than 80 regular Coulomb diamonds, illustrating that only one quantum dot is defined in this high quality carbon nanotube. The charging energy, $U \approx 5-6 \mathrm{meV}$ is estimated from the height of the diamonds and no clear shell structure is observed. ${ }^{27}$ A region of highly suppressed conductance around zero bias is clearly observed for all gate voltages reflecting the superconducting energy gap of the leads. Figure 1(b) shows the Coulomb diamonds in the dashed rectangle of Fig. 1(a). In Coulomb blockade (inside the diamonds) the onset of quasiparticle tunneling is seen as horizontal lines (conductance ridges) at $e V_{\mathrm{sd}}= \pm 2 \Delta$ $\approx \pm 0.55 \mathrm{meV}$ (horizontal green arrows). Higher-order Andreev reflections, which would lead to current below the gap, are strongly suppressed due to the poor coupling to the leads. Inside the diamonds, the onset of quasiparticle cotunneling corresponds to an alignment of the superconducting DOS peaks and involves elastic cotunneling processes as depicted in Fig. 1(c). At the charge-degeneracy points, conductance inside the gap is due to Andreev reflections. ${ }^{19}$ Figure 1(d) shows a bias cut slightly off-resonance (I) and further offresonance (II). The onset of quasiparticle tunneling at (I) involves a sequential tunneling process and is therefore much stronger than in (II). Peaks at higher bias are due to sequential tunneling to ground state (GS) and excited state (ES), respectively.

Figure 2(a) shows a bias spectroscopy plot for the second better coupled device (device $\mathrm{B}$ ) at $T=6.5 \mathrm{~K}$ well above the transition temperature $\left(T_{c} \approx 1.7 \mathrm{~K}\right)$ of the superconducting 

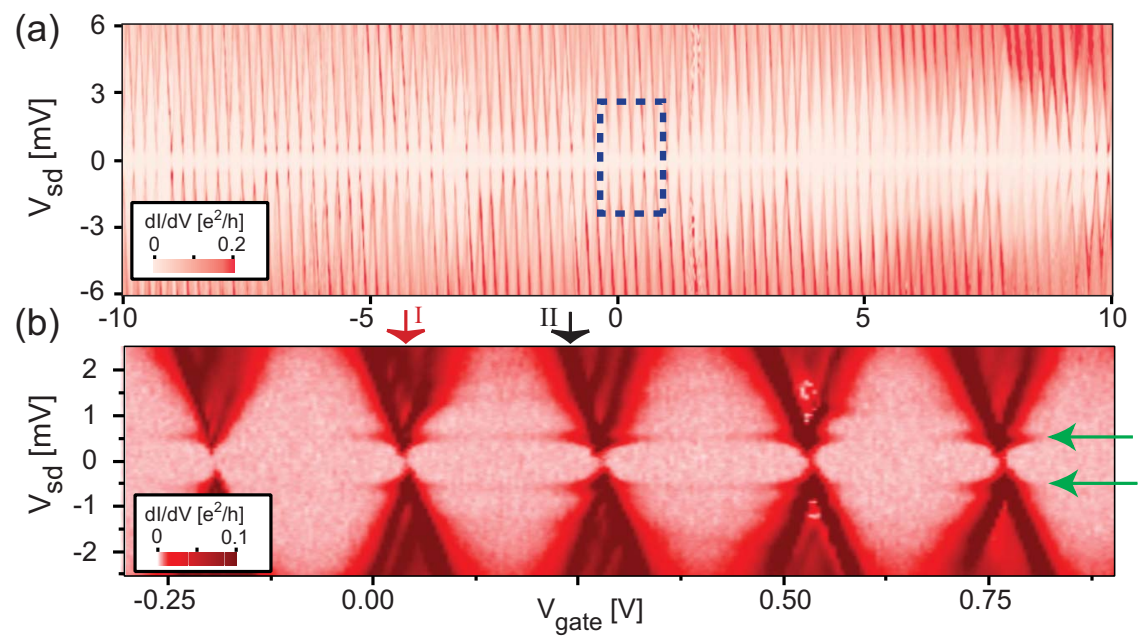

(c)

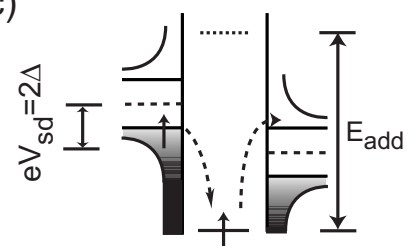

(d)

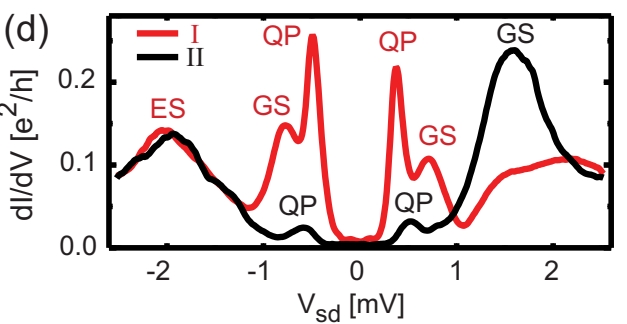

FIG. 1. (Color online) Device A. (a) Bias spectroscopy at $T$ $=0.3 \mathrm{~K}$ of a SWCNT coupled to $\mathrm{Ti} / \mathrm{Nb} / \mathrm{Ti}$ leads. (b) Conductance within the dashed rectangle in (a). The onset of quasiparticle cotunneling is clearly seen at $e V_{\text {sd }}$ $= \pm 2 \Delta$ (horizontal green arrows). (c) Schematic energy diagram for a viable cotunneling process in the center of a diamond. (d) Bias cuts at positions (I,II) in (b) showing conductance peaks due to QP cotunneling, and sequential tunneling into GS and ES, respectively.
$\mathrm{Pd} / \mathrm{Nb} / \mathrm{Pd}$ layer. A regular pattern is seen with three consecutive small Coulomb diamonds followed by a larger diamond reflecting the filling of shells consisting of two nearly degenerate orbitals. Numbers in Fig. 2 denote the additional electron number on the SWCNT for filled shells counted from $V_{\text {gate }} \approx-10 \mathrm{~V}$. A charging energy of $U \approx 12 \mathrm{meV}$ and a level spacing of $\Delta E \approx 6 \mathrm{meV}$ are found from the plot. Figure 2(b) shows the conductance at $T=0.3 \mathrm{~K}$, i.e., below $T_{c}$. The lines at $V_{\mathrm{sd}} \approx \pm 0.55 \mathrm{mV}$ (green horizontal arrows) are caused by elastic quasiparticle cotunneling as illustrated by Fig. 1(c).

Inelastic cotunneling lines [Fig. 2(b), blue arrows] are observed at higher $V_{\text {sd }}$ for electron numbers $N+1, N+2$, and $N+3$, but not in the full shell $(N+4$ electrons $)$. These lines have a marked gate voltage dependence which resembles the "double-headed arrow" structure pointed out in Ref. 4. With the enhanced spectroscopy offered by the superconducting leads we also observe an additional third "arrowhead" out-

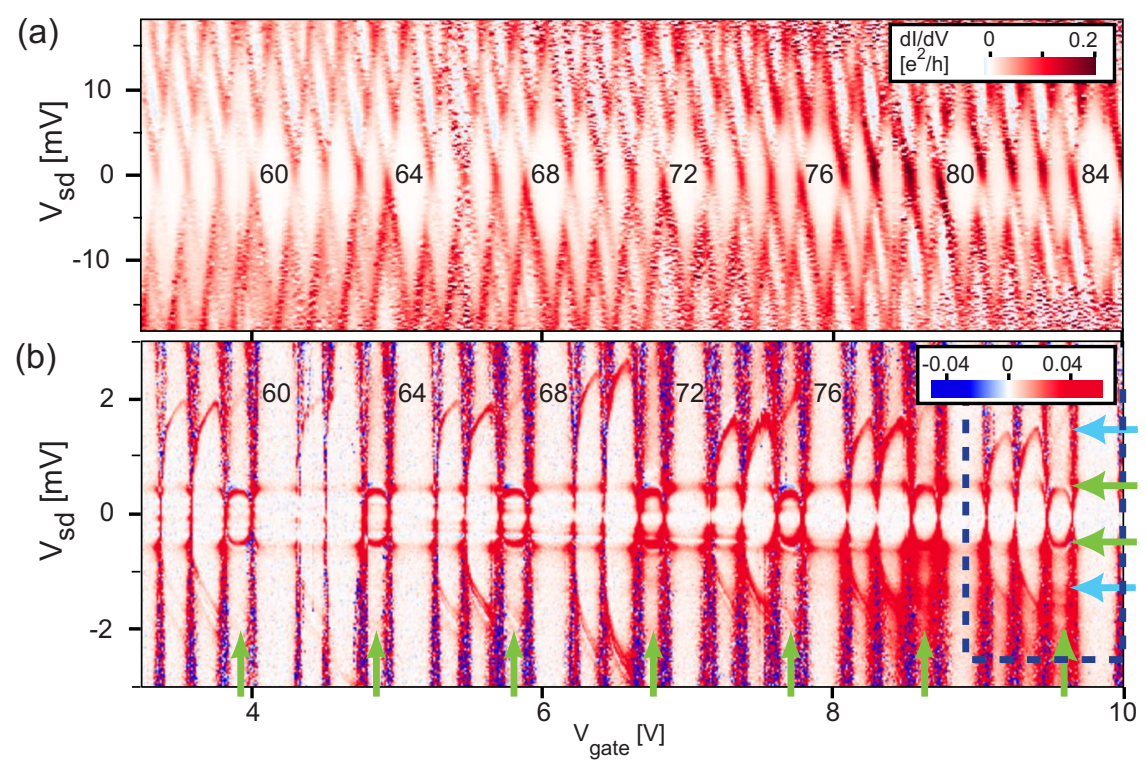

FIG. 2. (Color online) Device B. (a) Bias spectroscopy at $T=6.5 \mathrm{~K}$, i.e., above $T_{c} \approx 1.7 \mathrm{~K}$. Fourfold shell structure is observed with occupation numbers written in the large Coulomb diamonds corresponding to a full shell (zero occupation does not correspond to half filling). (b) Same gate region restricted to $\left|V_{\text {sd }}\right| \leq 3 \mathrm{mV}$ at $T=0.3 \mathrm{~K}$ showing inelastic cotunneling features (blue arrows) as well as the onset of elastic quasiparticle cotunneling (horizontal green arrows). Every fourth diamond shows pronounced gate-dependent conductance peaks near $\left|V_{\mathrm{sd}}\right|=2 \Delta / e$ (vertical green arrow). 


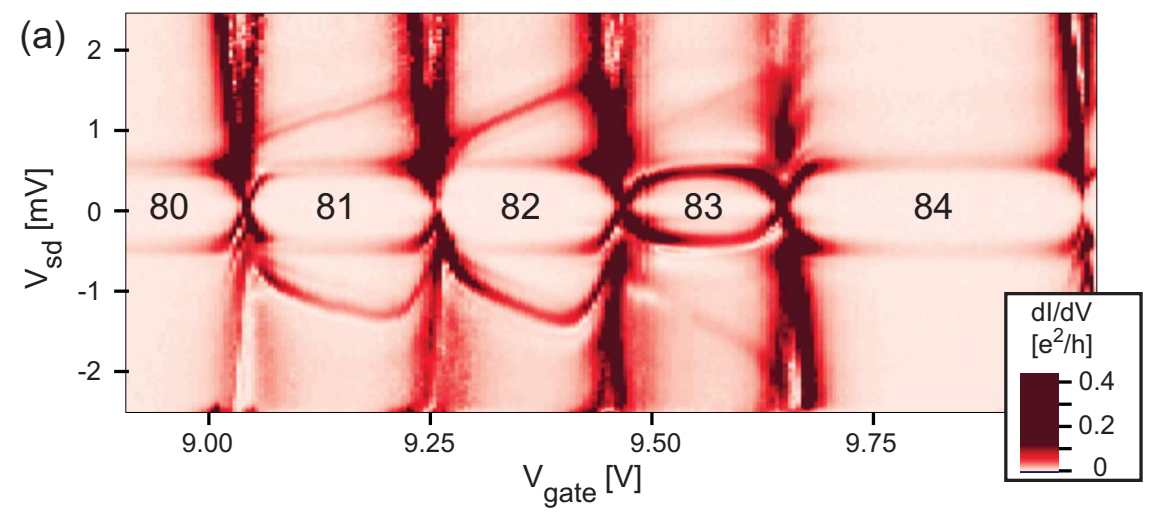

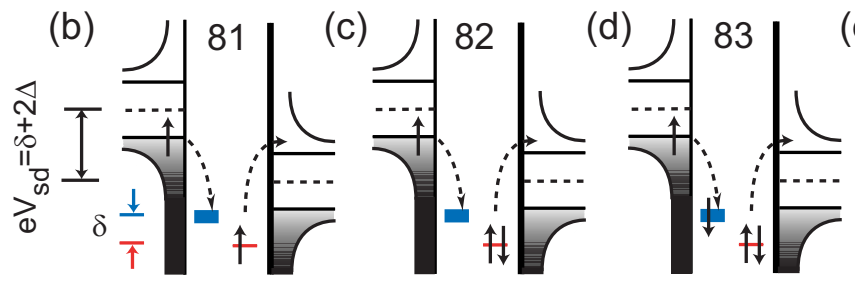

side the strong elastic cotunneling lines appearing in every fourth $(N+3)$ diamond, i.e., a possible Kondo ridge at this temperature in the normal state. The high $T_{c}$ and critical field of the $\mathrm{Nb}$ films prevented us from confirming the presence of a normal-state Kondo resonance, insofar as this resonance would already be suppressed by the magnetic field. The inelastic cotunneling lines are seen more clearly in Fig. 3(a) which shows detailed measurements from the dashed rectangle in Fig. 2(b). The cotunneling processes are depicted in Figs. 3(b)-3(e), involving a weaker coupled orbital (orbital 1) [thin red line in Fig. 3(b)] and a stronger coupled orbital (orbital 2) [thick blue line in Fig. 3(b)] split by $\delta$. Such processes are allowed for all but the charge state corresponding to a filled shell [Fig. 3(e)], consistent with Fig. 3(a). As

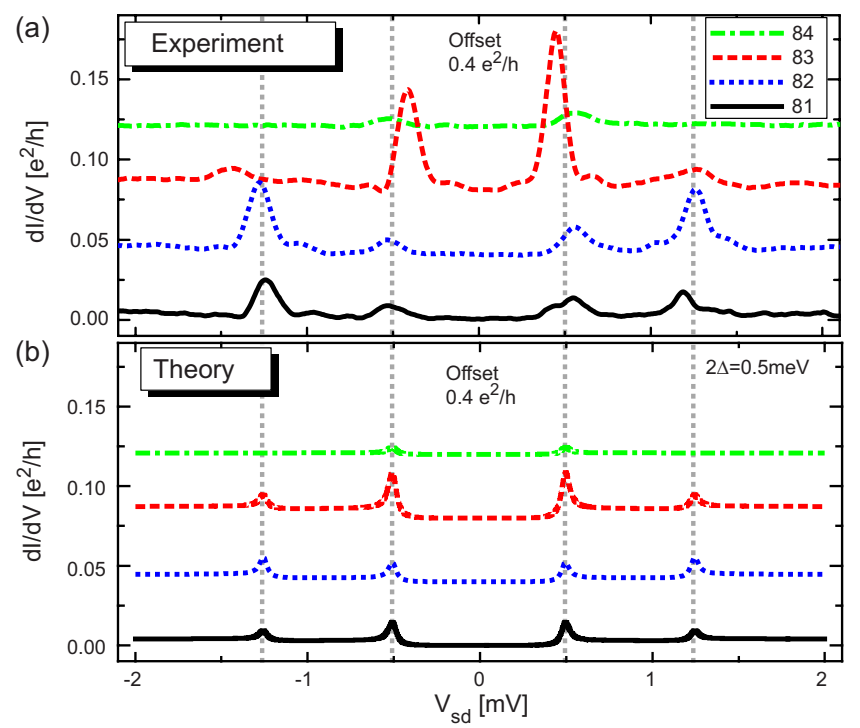

FIG. 4. (Color online) Comparison of (a) measured and (b) calculated conductance versus $V_{\text {sd }}$ in the center of diamonds 81-84 in Fig. 3(a). In the calculation $\delta=3 \Delta, k_{B} T=0.03 \mathrm{meV}$ and addition energies $(12.6,12.6,10.8$, and 20.7) $\mathrm{meV}$ extracted from the width of the diamonds $81-84$ have been used.
FIG. 3. (Color online) Device B. (a) Detailed bias spectroscopy at $T=0.3 \mathrm{~K}$ of one four-electron shell [dashed blue rectangle in Fig. 2(b)]. [(b)-(e)] Schematic energy diagrams illustrating inelastic cotunneling processes relevant for fillings of 1-4 electrons in a shell, e.g., corresponding to charge states $81-84$. The thick blue (thin red) level represents the strongly (weakly) coupled orbital, which both are coupled more weakly to the right electrode (drain) shown by a thicker barrier.

demonstrated by Holm et al., ${ }^{4}$ a difference in tunnel couplings to the two orbitals in the quantum dot gives rise to a gate dependence of the threshold for inelastic cotunneling. With superconducting leads, such tunneling renormalization produces a gate-dependent shift of the unrenormalized threshold at $e V_{\text {sd }} \geq \delta+2 \Delta$ as observed.

In Fig. 4(a) we show conductance line cuts through the center of each diamond $81-84$ in Fig. 3(a). The variation in the peak heights can be understood from the number of EL and INEL cotunneling channels in each diamond, as summarized in Table I. There, the EL notation indicates whether the tunneling takes place through the higher- or lower-energy orbital. For example, in case of filling 1 [see Fig. 3(b)], a total of six EL channels contribute: two from the upper and four from the lower orbital.

In Fig. 4(b) we show the results of a calculation of the lowest-order nonlinear cotunneling conductance for the four different charge states in a single shell. The calculation involves the quasiparticle tunneling rates $W_{i j}^{\alpha \beta}$ between leads $\alpha, \beta=L, R$ and orbitals $i, j=1,2$. For example, the rate for the process shown in Fig. 3(b) is

$$
W_{12}^{L R}=\frac{e}{h} \frac{4}{U^{2}} \int_{-\infty}^{\infty} d E \Gamma_{2}^{L}(E) \Gamma_{1}^{R}(E+\delta) f_{L}(E)\left[1-f_{R}(E+\delta)\right],
$$

with $f_{\alpha}(E)=f\left(E-\mu_{\alpha}\right)$ as the Fermi function, $\Gamma_{i}^{\alpha}(E)=\Gamma_{i}^{\alpha} \mid E$ $-\mu_{\alpha} \mid / \sqrt{\left(E-\mu_{\alpha}\right)^{2}-\Delta^{2}}$, and $\Gamma_{i}^{\alpha}=\pi \nu_{F}\left|t_{\alpha, i}\right|^{2}$ in terms of the tunneling amplitudes $t_{\alpha, i}$. Solving the steady-state rate equations we obtain the orbital occupation numbers as a function of $V_{\mathrm{sd}}$

TABLE I. Number of cotunneling channels.

\begin{tabular}{lcccc}
\hline \hline Shell filling & 1 & 2 & 3 & 4 \\
\hline EL & $\left(\begin{array}{c}2 \\
4\end{array}\right)$ & $\left(\begin{array}{c}2 \\
2\end{array}\right)$ & $\left(\begin{array}{c}4 \\
2\end{array}\right)$ & $\left(\begin{array}{c}2 \\
2\end{array}\right)$ \\
INEL & 2 & 4 & 2 & 0 \\
\hline \hline
\end{tabular}




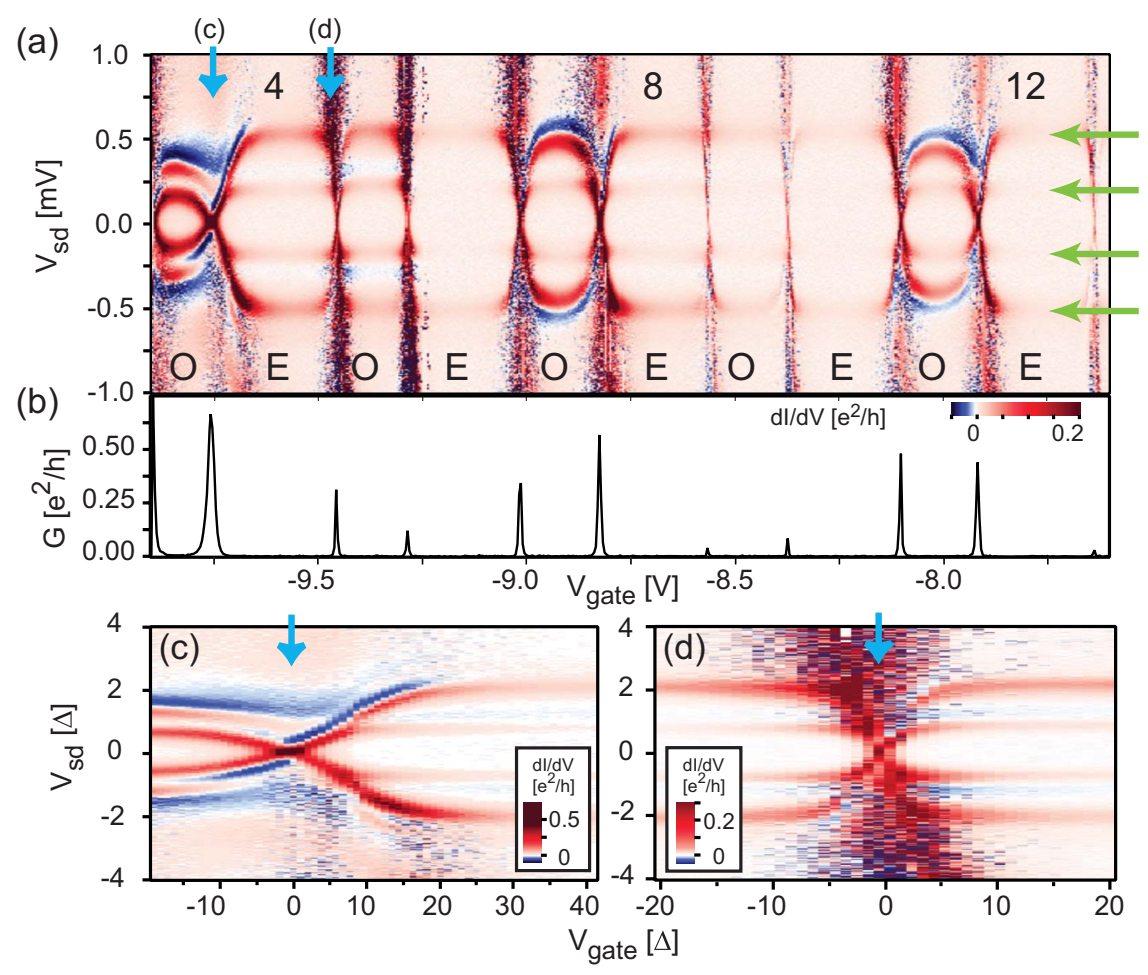

FIG. 5. (Color online) Device B. (a) Bias spectroscopy at $T=0.3 \mathrm{~K}$ for a strongly coupled gate region with pronounced change in the subgap structure in every fourth diamond. Numbers indicate the additional electron number in correspondence with Fig. 2, while the large/small diamonds are assigned even (E)/odd (O) electron filling. The horizontal green arrows indicate the elastic quasiparticle cotunneling lines at $e V_{\mathrm{sd}}= \pm 2 \Delta \approx \pm 0.5 \mathrm{meV}$ together with the lower-lying lines corresponding to a single Andreev reflection. (b) Linear conductance (zero bias) versus gate voltage hinting that well and poorly coupled orbitals are filled consecutively. [(c) and (d)] Zoom at the gap structure around two Coulomb blockade resonances marked by arrows in (a) with the gate voltage in units of $\Delta$.

and the current is readily determined. In agreement with experiment one sees from Fig. 4 that the inelastic cotunneling peak is largest for diamond 82 due to the larger number of tunneling possibilities. Likewise the elastic $2 \Delta$ peak is largest for diamond 83 due to the larger coupling of orbital 2 to the leads. The calculation does not reproduce the small shifts of some of the peaks seen in the experimental data, as well as the ratio between the amplitude of the elastic and inelastic peaks in diamond 81 . We speculate that the latter discrepancy will be removed by including higher-order processes involving the strongly coupled orbital 2 . The width of the peaks arise from a small smearing factor $(\eta=0.01 \mathrm{meV})$ used in the DOS of the superconducting leads mimicking a constant inelastic scattering rate in the contacts. We ascribe the larger width of the measured peaks to tunnel broadening of the excited states which is not included in the calculation.

For the results shown in Fig. 4 we have used $\Gamma_{1}^{R}=0.07 \mathrm{meV}, \quad \Gamma_{2}^{R}=0.1 \mathrm{meV}, \quad \Gamma_{1}^{L}=1.0 \mathrm{meV}$, and $\Gamma_{2}^{L}$ $=1.8 \mathrm{meV}$, yielding an asymmetry factor in the order of $\Sigma_{i} \Gamma_{i}^{L} / \Sigma_{i} \Gamma_{i}^{R} \approx 15$ consistent with an upper bound ${ }^{28}$ of approximately 40 , extracted from the Coulomb peak heights in the shell at $6.5 \mathrm{~K}$. The couplings are estimated from the sequential current at large positive $\left(I^{+}\right)$and negative biases $\left(I^{-}\right)$at the charge-degeneracy point for adding the first electron in a fourfold degenerate shell. These currents are given by $I^{+/-}=\frac{e}{h} \frac{2 \Gamma_{1}^{L / R} \Gamma_{2}^{L / R}\left(\Gamma_{1}^{R / L}+\Gamma_{2}^{R / L}\right)}{2\left(\Gamma_{2}^{L / R} \Gamma_{1}^{R / L}+\Gamma_{1}^{L / R} \Gamma_{2}^{R / L}\right)+\Gamma_{1}^{L / R} \Gamma_{2}^{L / R}} \approx 11 \mathrm{nA} /-3.5 \mathrm{nA}$ in reasonable agreement with the experiment at the Coulomb reso- nance involving electron charge states 80 and 81, $I_{\exp }^{+/-} \approx 12 \mathrm{nA} /-3 \mathrm{nA}$. Moreover, the chosen couplings lead to a gate-voltage slope ${ }^{4}$ of the inelastic $2 \Delta+\delta$ line of $d \delta / d\left(e \gamma V_{g}\right)=4 \Sigma_{\alpha}\left(\Gamma_{2}^{\alpha}-\Gamma_{1}^{\alpha}\right) / \pi U \approx 0.084$ (average $U$ $=12.0 \mathrm{meV}$ ), which agrees with the experimental result of 0.082 [average slope of the cotunneling lines in diamonds 81-83 in Fig. 3(a)]. We note that since $U \gg \Delta$, the expression for the renormalization obtained in the normal state ${ }^{4}$ remains valid in the case of superconducting leads. Based on the couplings $\Gamma_{i}^{\alpha}$ used above, we estimate the Kondo energies (temperatures) in diamonds 81 and 83 to be $k_{B} T_{K, 81}$ $\approx 0.2 \mu \mathrm{eV}(2 \mathrm{mK})$ and $k_{B} T_{K, 83} \approx 0.03 \mathrm{meV}(300 \mathrm{mK})$, i.e., much smaller than $\Delta \sim 0.28 \mathrm{meV}$, consistent with our observation of subgap structure rather than an enhanced zero-bias conductance peak. ${ }^{13}$

We now discuss measurements from a better coupled gate voltage region of device $\mathrm{B}$, exhibiting a characteristic rounding of the elastic quasiparticle cotunneling and an unusual subgap conductance as seen in Fig. 5(a). For charge states with three electrons in a shell, the subgap structure is especially pronounced and gate dependent, indicating that this orbital is particularly well coupled to the leads. This is supported by the linear conductance data presented in Fig. 5(b), showing broad resonances where the effect is largest (diamonds 3,7 , and 11). In diamond 3 , the subgap conductance even exceeds the elastic cotunneling peak in the other diamonds at $e V_{\text {sd }} \sim \pm 2 \Delta,{ }^{17,18}$ with strong peaks at voltages which are different from the expected MAR positions at 
$\pm 2 \Delta / n$. Figure 5(a) also displays marked negative differential conductance, seen as blue lines reflecting a local minimum in the current at $e V_{\mathrm{sd}} \sim \pm 2 \Delta$ in the center of diamonds 3,7 , and 11. A similar negative differential conductance effect has been reported in a nanotube quantum dot with very different couplings to source and drain electrodes. ${ }^{17}$

In Figs. 5(c) and 5(d) we show a zoom-in of two neighboring charge-degeneracy points $(N=3 / 4$ and $N=4 / 5)$ marked by vertical (blue) arrows in Fig. 5(a). Panel (d) shows a close resemblance to the behavior expected from MAR in the presence of a resonant level $(\Gamma \lesssim \Delta)$ (Refs. 19, 24 , and 25) with the rounding of the subgap structure extending some $4 \Delta$ into the Coulomb blockade diamond. The data in panel (c), on the other hand, exhibit a qualitatively different subgap structure with pronounced negative differential conductance and a much stronger gate dependence which levels off at roughly $15-20 \Delta$ away from the chargedegeneracy point.

We speculate that the unusual subgap features observed in odd occupied dots are caused by an interplay between MAR and quantum Shiba states, ${ }^{26}$ present for spinful dots with $k_{B} T_{K}<\Delta$. For strongly asymmetric couplings, these spininduced bound states remain pinned to the stronger coupled lead at energies inside the gap given roughly by the exchange coupling with this electrode. Therefore, new conductance peaks away from the usual $\pm 2 \Delta / n$ occur naturally in this scenario, and a bias scan with the coherence peaks of the weaker coupled lead gives rise to negative differential conductance at $\pm 2 \Delta$ because of spectral weight transfer from the coherence peaks of the stronger coupled lead to the bound states.

In summary, we have demonstrated how superconducting electrodes lead to dramatic enhancement of cotunneling spectroscopy in carbon nanotube quantum dots. This revealed pronounced inelastic cotunneling lines with marked gate dependence caused by tunneling-induced level shifts. Moreover, we discussed the presence of negative differential conductance and unusual subgap conductance in strongly coupled odd-occupied diamonds. Further studies are required to fully uncover the interesting interplay between MAR and spin correlations in quantum dots.

We acknowledge experimental help from Jørn Bindslev Hansen. This work was supported by V. K. Rasmussen Foundation, Danish Agency for Science, Technology and Innovation, Carlsberg Foundation, CARDEQ and SECOQC projects, and the Danish Research Council.
*Present address: NTT Corporation, NTT Basic Research Laboratories, 3-1 Morinosato Wakamiya, Atsugi-shi, 243-0198 Kanagawa, Japan; grove@will.brl.ntt.co.jp

${ }^{1}$ E. L. Wolf, Principles of Electron Tunneling Spectroscopy (Oxford University Press, New York, 1985).

${ }^{2}$ I. Giaever, Phys. Rev. Lett. 5, 464 (1960).

${ }^{3}$ D. C. Ralph, C. T. Black, and M. Tinkham, Phys. Rev. Lett. 74, 3241 (1995).

${ }^{4}$ J. V. Holm, H. I. Jørgensen, K. Grove-Rasmussen, J. Paaske, K. Flensberg, and P. E. Lindelof, Phys. Rev. B 77, 161406(R) (2008).

${ }^{5}$ A. Y. Kasumov, R. Deblock, M. Kociak, B. Reulet, H. Bouchiat, I. I. Khodos, Y. B. Gorbatov, V. T. Volkov, C. Journet, and M. Burghard, Science 284, 1508 (1999).

${ }^{6}$ T. Tsuneta, L. Lechner, and P. J. Hakonen, Phys. Rev. Lett. 98, 087002 (2007).

${ }^{7}$ E. Pallecchi, M. Gaaß, D. A. Ryndyk, and C. Strunk, Appl. Phys. Lett. 93, 072501 (2008).

${ }^{8}$ A. F. Morpurgo, J. Kong, C. M. Marcus, and H. Dai, Science 286, 263 (1999).

${ }^{9}$ P. Jarillo-Herrero, J. A. van Dam, and L. P. Kouwenhoven, Nature (London) 439, 953 (2006)

${ }^{10}$ H. I. Jørgensen, K. Grove-Rasmussen, T. Novotný, K. Flensberg, and P. E. Lindelof, Phys. Rev. Lett. 96, 207003 (2006).

${ }^{11}$ J.-P. Cleuziou, W. Wernsdorfer, S. Andergassen, S. Florens, V. Bouchiat, T. Ondarcuhu, and M. Monthioux, Phys. Rev. Lett. 99, 117001 (2007).

${ }^{12}$ Y. Zhang, G. Liu, and C. N. Lau, Nano Res. 1, 145 (2008).

${ }^{13}$ M. R. Buitelaar, T. Nussbaumer, and C. Schönenberger, Phys. Rev. Lett. 89, 256801 (2002).

${ }^{14}$ J.-P. Cleuziou, W. Wernsdorfer, V. Bouchiat, T. Ondarçuhu, and M. Monthioux, Nat. Nanotechnol. 1, 53 (2006).

${ }^{15} \mathrm{~K}$. Grove-Rasmussen, H. I. Jørgensen, and P. E. Lindelof, New J.
Phys. 9, 124 (2007)

${ }^{16}$ C. Buizert, A. Oiwa, K. Shibata, K. Hirakawa, and S. Tarucha, Phys. Rev. Lett. 99, 136806 (2007).

${ }^{17}$ A. Eichler, M. Weiss, S. Oberholzer, C. Schönenberger, A. Levy Yeyati, J. C. Cuevas, and A. Martín-Rodero, Phys. Rev. Lett. 99, 126602 (2007).

${ }^{18}$ T. Sand-Jespersen, J. Paaske, B. M. Andersen, K. GroveRasmussen, H. I. Jørgensen, M. Aagesen, C. B. Sørensen, P. E. Lindelof, K. Flensberg, and J. Nygård, Phys. Rev. Lett. 99, 126603 (2007).

${ }^{19}$ M. R. Buitelaar, W. Belzig, T. Nussbaumer, B. Babić, C. Bruder, and C. Schönenberger, Phys. Rev. Lett. 91, 057005 (2003).

${ }^{20}$ H. I. Jørgensen, T. Novotný, K. Grove-Rasmussen, K. Flensberg, and P. E. Lindelof, Nano Lett. 7, 2441 (2007).

${ }^{21}$ J. A. van Dam, Y. V. Nazarov, E. P. A. M. Bakkers, S. De Franceschi, and L. P. Kouwenhoven, Nature (London) 442, 667 (2006).

${ }^{22}$ Y.-J. Doh, S. De Franceschi, E. P. A. M. Bakkers, and L. P. Kouwenhoven, Nano Lett. 8, 4098 (2008).

${ }^{23}$ J. Hulm, C. Jones, R. Hein, and J. Gibson, J. Low Temp. Phys. 7, 291 (1972).

${ }^{24}$ A. L. Yeyati, J. C. Cuevas, A. López-Dávalos, and A. MartínRodero, Phys. Rev. B 55, R6137 (1997).

${ }^{25}$ G. Johansson, E. N. Bratus, V. S. Shumeiko, and G. Wendin, Phys. Rev. B 60, 1382 (1999)

${ }^{26}$ T. Soda, T. Matsuura, and Y. Nagaoka, Prog. Theor. Phys. 38, 551 (1967).

${ }^{27} \mathrm{~A}$ variation in the addition energies is seen, but no clear fourelectron shell structure is observed as is the case of device B. The origin of this difference is not understood except that the energy scales related to device B are much larger than in device A, making such effects more visible.

${ }^{28}$ The Coulomb peaks measured at $T=6.5 \mathrm{~K}$ are already slightly suppressed by temperature. 